Discussion MSM in our centre who decline HIV testing are more likely to report recent/ever testing and around $40 \%$ of all new attendees report a previous HIV test. It is of concern that patients who refuse testing have higher rate of STI diagnoses increasing their likelihood of HIV acquisition. The outcome of this audit is to re-visit our documentation of reasons for declining HIV testing and re-inforce strategies to improve uptake in this group

\section{P179 CHARACTERISTICS OF MSM ATTENDEES AND RATE OF STI RE-TESTING IN A DEDICATED MSM SERVICE VERSUS THE GENERAL WALK IN GU CLINIC}

Mauro Proserpio, Olamide Dosekun*. Imperial College Healthcare NHS Trust, London, UK

10.1136/sextrans-2016-052718.229

Background Men who have sex with men (MSM) in London experience inequalities in sexual health despite bearing the highest burden of sexually transmitted infections (STIs). Annual STI screening in MSM is recommended in the UK. Our London based clinic has a dedicated service for MSM to foster rapport with patients, promote safer sex and normalise regular STI screening.

Aims To describe characteristics of MSM attending a general walk in clinic (GWI) versus a dedicated MSM service (MSMS), and rate of STI retesting between the two clinics.

Methods A case-note review of MSM and bisexual male attendees between October and December 2014 in the GWI and MSMS and new episode attendances up to January 2016.

Results Information on 101 MSM (50 GWI, 51 MSMS) was collected; median age was 32 (range 25-59) and 29 (22-49) years respectively, and they were of white ethnicity in $33 / 50,66 \%$, and 40/51, 78\% in GWI and MSMS respectively. There were no differences in HIV status or new STI/HIV diagnoses between clinic attendees. There were 82 and 104 further new episode attendances amongst these GWI and MSMS attendees respectively; the latter mostly re-attended the MSMS (69/101, 68\%). There was no differences in number of non-reattenders (18, 36\%; 19, 37\% respectively).

Conclusion Patients in our MSMS tended to re-attend more for repeat screening and within the same service, in comparison to GWI. Similar numbers of patients never re-attended in both clinics, suggesting that further strategies are needed to embed a culture of regular screening in this group.

\section{P180 AN UNUSUAL CAUSE OF PHARYNGO-TONSILLAR ULCERATION}

Siew Yan Teo, Cara Saxon*. Bridgewater Community Healthcare NHS Foundation Trust, Manchester, UK

10.1136/sextrans-2016-052718.230

Background/introduction Herpes Simplex 1 virus has historically been known to cause oral and genital symptoms, whereas Herpes Simplex 2 virus is mostly associated with genital symptoms. We present the first case in the UK, to our knowledge, of primary Herpes Simplex 2 virus causing genital and pharyngo-tonsillar ulceration in a sexually active female patient.
Case A 33 year old female patient attended the GUM clinic reporting 2 day history of genital sores associated with dysuria. She has recently completed a 3 day course of Nitrofurantoin for presumed UTI with no effect, and is currently taking a course of Penicillin for tonsillitis. She has a new male partner of 1 month duration. Genital examination revealed bilateral inguinal lymphadenopathy with multiple herpetic lesions on the labia majora and minora. Pharyngeal examination revealed pustular looking tonsils with ulceration bilaterally, more marked on the left. Cervical chain lymphadenopathy was also present. HSV PCR swabs taken from both the tonsillar and genital ulcers came back positive for HSV-2. She was initially treated with a 10 day course of Aciclovir and returned for follow up 1 week later. Repeat examination revealed fully healed vulval ulcers and normal tonsillar appearance.

Discussion/conclusion This is the first UK reported case of primary HSV-2 causing pharyngo-tonsillar ulceration in addition to genital symptoms. The patient made a full and rapid recovery following prompt treatment with Aciclovir. This case highlights the importance of recognising less common causes for tonsillitis such as HSV-2, which responds very quickly to antiviral treatment.

\section{P181 DO WE NEED TO ROUTINELY RE-SCREEN HETEROSEXUAL PATIENTS FOR HIV AND SYPHILIS WHEN THEY REATTEND GUM/SRH CLINICS? (IMPROVING CLINICAL PRACTICE AND SERVICE DELIVERY)}

Nilani Uthayakumar*, Eva Jungmann. Mortimer Market Centre, London, UK

\subsection{6/sextrans-2016-052718.231}

Background/introduction In the current financial climate we need to ensure that scarce resources are used efficiently. Heterosexual patients re-attending GUM/SRH services after an initial negative screen for HIV may be at low risk for acquiring new HIV and/or Syphilis and routine testing may not be costeffective.

Aim(s)/objectives Determine the incidence of HIV and Syphilis in heterosexuals re-attending GUM/SRH services after previous negative HIV testing. Establish potential savings.

Methods Case note review of heterosexual patients attending a mixture of Inner London Integrated and Sexual Health clinics in 2014 and re-testing for HIV and/or Syphilis within 12 months. Data extracted includes patient demographics, tests performed and outcomes. Cost of HIV POCT $£ 2.64$, HIV serology $£ 18.75$, Syphilis screen $£ 10.35$.

Results Of 31,469 patients who tested for HIV in 2014, 4,584 $(14.6 \%)$ were retested within 12 months. 69\% were female and $31 \%$ male. The age range was $16-81$ years with $27 \%<25$ years, 33\% White British, 20\% White Other, 18\% Black African/Black British/Caribbean/Other Black and others from a diverse range of ethnicities. 89\% tested for HIV (82\% POCT, 18\% Antigen/ Antibody) and $88 \%$ for Syphilis. Results showed one newly diagnosed HIV infection (male seroconverter with recent high-risk activity) and no new Syphilis infections. 25 patients found with positive Syphilis Serology were all either previously treated or had a false positive result. Potential savings if we had not tested for: HIV POCT- $£ 8,886$, HIV Antigen/Antibody- $£ 13,763$, Syphilis- $£ 42,083$. 